\title{
High Levels of Genetic Diversity within One Population of Rheum tanguti- cum on the Qinghai-Tibet Plateau have Implications for Germplasm Conservation
}

\author{
Xiaochong $\mathrm{Ma}^{1}$, Caixiang Xie ${ }^{1}$, Meng Guan ${ }^{2}$, Xiuhai Xu ${ }^{2}$, Eiji Miki ${ }^{3}$, Osami Takeda ${ }^{3}$, Tianyi Xin ${ }^{1}$, \\ Sihao Zheng ${ }^{1}$, Hui Yao ${ }^{1}$, Linchun Shi ${ }^{1}$, Jingyuan Song ${ }^{1, *}$ and Shilin Chen ${ }^{1}$ \\ ${ }^{1}$ Institute of Medicinal Plant Development, Chinese Academy of Medical Sciences, Peking Union Medical College, 151 \\ Malianwa North Road, Haidian District, Beijing 100193, China ${ }^{2}$ China Meheco Corporation, Beijing 100061, China; \\ ${ }^{3}$ Botanical Raw Material Research Department, TSUMURA \& CO, Japan
}

\begin{abstract}
Rheum tanguticum, a source plant for the traditional Chinese medicine Dahuang (rhubarb), is an endangered species. In this study, we first assessed genetic diversity using 102 internal transcribed spacer (ITS) sequences cloned from one population of $R$. tanguticum on the Qinghai-Tibet Plateau. In total, 1773 polymorphic sites were identified, including 707 sites in the ITS1 region, 773 sites in the ITS2 region, and 293 sites on the 5.8S gene. Overall, transitions were the most common type of mutation and represented $77 \%$ of all base substitutions found within the ITS regions of $R$. tanguticum. We found that the average intra-specific genetic distance was $0.1260 \pm 0.0755$ and ranged from 0 to 0.3618 . Furthermore, a neighbor-joining phylogenetic tree constructed using these sequences strongly supported the conclusion that abundant intra-specific variation exists within the ITS regions of $R$. tanguticum. Interestingly, we found that eightyseven clones of $R$. tanguticum had high similarity to the ITS sequence of $R$. rhabarbarum and ten clones had high similarity to the ITS sequence of $R$. officinale; however, five clones of $R$. tanguticum had similarity to Fagopyrum esculentum. Therefore, we observed a significant amount of ITS sequence variation at both the intra-genomic and intra-specific levels in $R$. tanguticum. Based on these results, we suggest that in situ conservation is preferable for $R$. tanguticum protection.
\end{abstract}

Keywords: Genetic diversity, intra-specific variation, Rheum tanguticum, internal transcribed spacer, endangered species conservation.

\section{INTRODUCTION}

Rheum tanguticum Maxim. Ex Balf. (Polygonaceae) is one of the three main sources of Dahuang (rhubarb), a wellknown traditional Chinese medicine (TCM). Its phenolic compounds and polysaccharides have shown some promising pharmacological properties [1,2]. The dried rhizomes and roots of $R$. tanguticum have been widely used as laxatives and anti-inflammatory agents in China [3], while the standardized rhubarb extracts, such as emodin, have become available on the international market $[4,5]$. R. tanguticum, also known as Tangut rheumor chicken-feet rheum, is native to the Qinghai-Tibet Plateau at altitudes ranging from 2300 to $4200 \mathrm{~m}[6,7]$. In recent years, the wild resources of $R$. tanguticum were seriously threatened by over-exploitation and an extensive loss of habitat; thus, this species has been included on the important conservation of wild plants list for the Qinghai province [8]. Some measures have been taken to reduce the difference between the commercial demand and the wild resource depletion, such as cultivation in Guoluo [9]. However, reductions to wild $R$. tanguticum populations are a major concern for development of superior

\footnotetext{
*Address correspondence to this author at the Institute of Medicinal Plant Development, Chinese Academy of Medical Sciences, Peking Union Medical College, 151 Malianwa North Road, Haidian District, Beijing 100193, China; Tel: 86-10-57833199; Fax: 86-10-62895907;

Email: jysong@implad.ac.cn
}

pharmaceutical crop cultivars of the Tangut rheum. Analysis of genetic variations in the endangered species at the population level is critically important for conservation strategies because sufficient genetic variation can increase the species chance of survival [10]. Therefore, the analysis of genetic variation within and between populations becomes important not only for the conservation of the endangered species [1115] but also for pharmaceutical crop development.

The internal transcribed spacer (ITS) of nuclear ribosomal DNA (nrDNA) has been used extensively in studies on genetic diversity, phylogenetics, and evolution at the species or genera levels due to its fast rate of evolution and great variation [16-18]. The ITS region is composed of three parts: ITS1, ITS2, and the highly conserved 5.8S rDNA gene, which islocated between ITS1 and ITS2 [19]. Compared to organellar DNA, the ITS of nrDNA, which is inherited from both parents, can provide much more information [20]. Furthermore, this region is simple to amplify using PCR and a set of universal primers, which are available for a wide range of organisms, and it can be easily sequenced due to its relatively small size [21]. Importantly, high levels of variation are usually observed within ITS regions and are ideal for revealing genetic diversity and population differentiation in many species [22-24]. However, to date, there have been no reports of ITS sequence variation at the intra-specific level in $R$. tanguticum. In previous reports, genetic diversity of $R$. tanguticum was revealed based on ISSR and SSR analysis 
$[8,25]$. These studies demonstrated most of genetic variation within populations of $R$. tanguticum rather than between.

The goals of the present study were to identify and characterize the distribution of sequence variation based on the ITS sequences within one population of $R$. tanguticum, analyze potential reasons for sequence variations, and propose conservation strategies for this species.

\section{MATERIALS AND METHODOLOGY}

\section{Plant Materials}

Twenty-nine specimens of $R$. tanguticum were collected from the Baihe pasture of Zoige in the Sichuan Province of China (latitude: $33^{\circ} 18^{\prime} 35^{\prime \prime} \mathrm{N}$ to $33^{\circ} 21^{\prime} 32^{\prime \prime} \mathrm{N}$; longitude: $102^{\circ} 27^{\prime} 12^{\prime \prime} \mathrm{E}$ to $102^{\circ} 28^{\prime} 9^{\prime \prime} \mathrm{E}$; altitude: $3436-3459 \mathrm{~m}$ ) (see the Appendix Table S1). A typical habitat for $R$. tanguticum is shown in (Fig. 1).

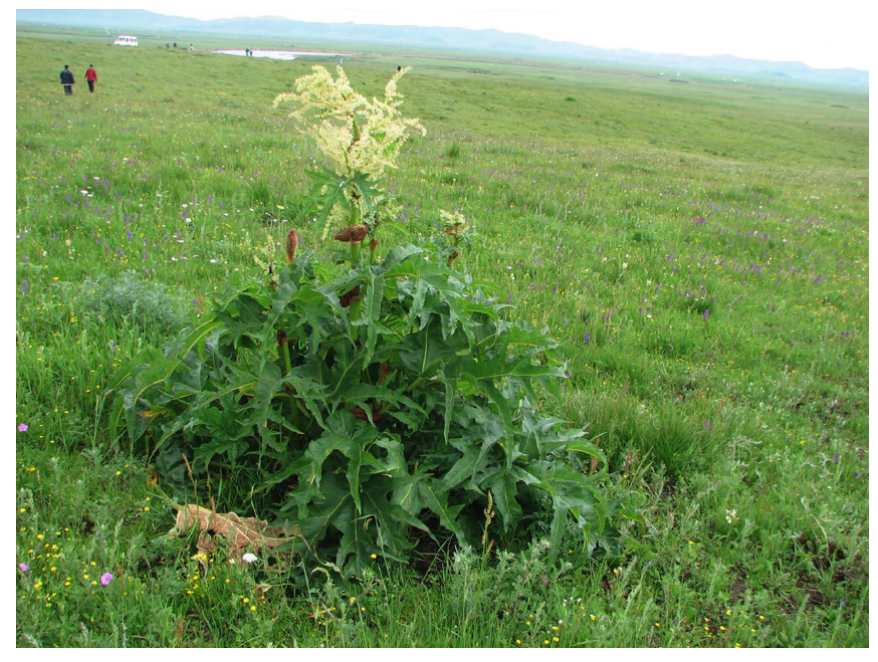

Fig. (1). The habitat of Rheum tanguticum.

\section{Genomic DNA Preparation and PCR Amplification}

Genomic DNA was extracted from $20 \mathrm{mg}$ of silica geldried leaves using the Plant Genomic DNA Kit (Tiangen Biotech Co., China) after grinding for $1 \mathrm{~min}$ at 30 revolutions/s with a DNA Extraction Grinder (MM400; Retsch, Haan, Germany). Polymerase chain reaction (PCR) amplifications of the ITS region were carried out using a Peltier Thermal Cycler ABI9700 (ABI lab, Inc., USA) and the following pair of universal primers: ITS5F (5'GGAAGTAAAAGTCGTAACAAGG-3') and ITS4R (5'TCCTCCGCTTATTGATATGC -3') [26]. PCR amplification was performed using 20-50 ng of genomic DNA as template and a total volume of $25 \mu \mathrm{L}$, which also contained the following: $1 \times \mathrm{PCR}$ buffer (without $\mathrm{MgCl}_{2}$ ), $2.0 \mathrm{mM} \mathrm{MgCl}$, $0.2 \mathrm{mM}$ each dNTP, $0.1 \mu \mathrm{M}$ each ITS primer (synthesized by Sangon Co., China), and 1.0 U Taq DNA Polymerase (Biocolor BioScience \& Technology Co., China). PCR reaction conditions consisted of an initial denaturation step for $5 \mathrm{~min}$ at $94^{\circ} \mathrm{C}$, followed by 35 cycles of denaturation for $1 \mathrm{~min}$ at $94^{\circ} \mathrm{C}$, annealing for $1 \mathrm{~min}$ at $50^{\circ} \mathrm{C}$ and extension for $1.5 \mathrm{~min}$ at $72^{\circ} \mathrm{C}(+3 \mathrm{~s} /$ cycle $)$, with a final extension step at $72^{\circ} \mathrm{C}$ for 7 min [27]. The PCR products were separated on $1.0 \%$ agarose gels in $0.5 \times \mathrm{TBE}$ buffer for visualization. Direct sequencing of the PCR products from $R$. tanguticum was unsuccessful, indicating the presence of polymorphisms within individual specimens.

\section{Cloning and Sequencing of the ITS Region}

The PCR products were purified using the TIAN quick Midi Purification Kit (Tiangen Biotech Co., China). The recovered products were ligated into the pMD19-T vector (Takara Biotech Co., China) following the addition of dATP residues to the 3' ends of the PCR products using the DNA A-Tailing Kit (Takara Biotech Co., China). The cloned products were then transformed into $E$. coli $\mathrm{DH} 5 \alpha$ cells using standard recombinant DNA techniques.

E. coli colonies were cultured in LB culture medium containing ampicillin, and following selection with colony PCR, five positive clonesfrom each sample were sequenced using an ABI3730XL sequencer (Majorbio Co., Shanghai, China). In total, we obtained 105 clones from 29 samples (excluding fungal sequences). Three of the clones (including KF514611, KF514546 and KF514613) showed significant differences when compared with the other sequences ( $\sim 90 \mathrm{bp}$ deletions at the start of the ITS region) and were not analyzed in this paper (see the sequence alignment in Appendix Fig. S1, S2, and $\mathbf{S 3}$ ).

\section{Sequence Alignment and Date Analysis}

The sequences of the ITS regions of $R$. tanguticum were aligned using Codoncode Aligner3.7.1 (CodonCode Co., USA). Genetic variations were analyzed with a Kimura 2parameter (K2P) distance matrix, which was constructed using the MEGA5.0 software program [28]. The neighborjoining (NJ) phylogenetic trees were built in the MEGA5.0 software program using the 102 sequenced clones from $R$. tanguticum as well as four other GenBank sequences from other species: Rheum officinale (JN187108), Rheum nobile (GQ206264), Rheum rhabarbarum (JQ288756), and Fagopyrum esculentum (EF653685). The 5.8S gene and the ITS1 region were obtained through sequence alignment with $R$. rhabarbarum and $F$. esculentum, and the ITS2 region was obtained using hidden Markov model (HMM)-based annotation methods [29].

\section{RESULTS}

\section{Sequence Length and GC Content}

The average and distribution of the ITS sequence lengths, as well as GC contents, are shown in (Fig. 2). The ITS sequence length ranged from 522-571 bp in the 102 clones from $R$. tanguticum. The average length of the ITS sequences was $559 \mathrm{bp}$, and the median length was similar to the average. The GC content of the ITS sequences from $R$. tanguticum ranged from 56- 69\%, with an average value of $63 \%$ and a median of $62 \%$.

\section{Sequence Variation and Polymorphic Distributions of the ITS Region within Individual Genomes}

We did not analyze insertions and deletions (indels) in the present study, as they are often spread across multiple sites, particularly in long homopolymeric tracts, and are difficult to identify in an accurate and reliable manner. Therefore, we limited ourselves to the detection and comparative 
analysis of base substitution-only type polymorphisms (e.g., transitions and transversions).

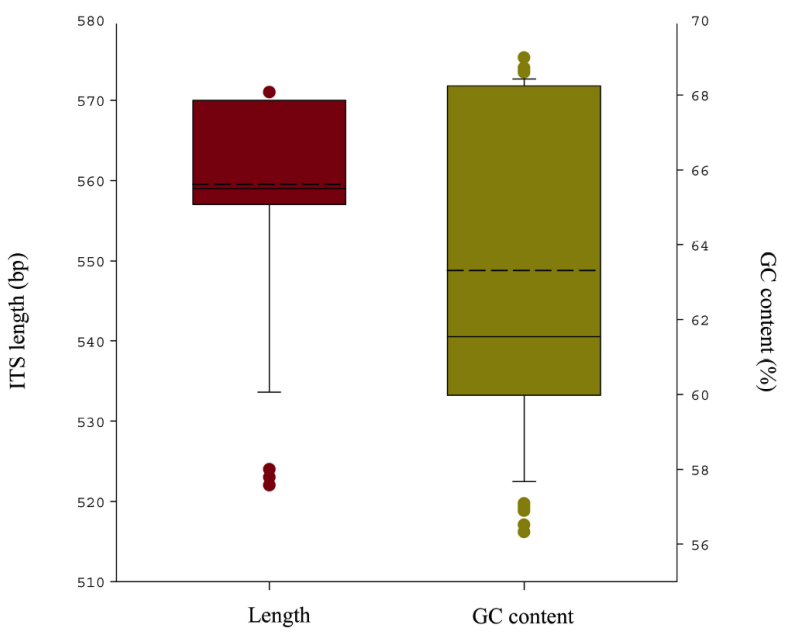

Fig. (2). ITS sequence length and GC content in 102 clones of Rheum tanguticum. Box plots show the interquartile range (IQR) of the data, which is defined as the difference between the 75th and 25 th percentiles. The solid and dotted lines in the box represent the median and average values, respectively, for length and GC content.

Despite the exclusion of indels from our study, we identified a remarkably high level of intra-individual variation in $R$. tanguticum, ranging from 0 (in the sample RTM02) to 168 polymorphisms (in the sample RTM09). In total, 1773 polymorphic sites were identified: 707 sites in the ITS1 region, 773 sites in the ITS2 region, and 293 sites on the $5.8 \mathrm{~S}$ exon (Fig. 3). Overall, the ITS2 regions had nearly one fold more polymorphisms than the ITS1 region. Within the ITS1 region, the polymorphisms were evenly distributed over the $200 \mathrm{bp}$, whereas polymorphisms in ITS2 were primarily found in the 350-550 bp regions. Transversions were detected at the highest frequency in the 500-550 bp region of ITS2. Furthermore, we found transitions to be the most common type of mutation, representing 77\% (1366 of 1773 sites) of all base substitutions in the ITS regions of $R$. tanguticum.

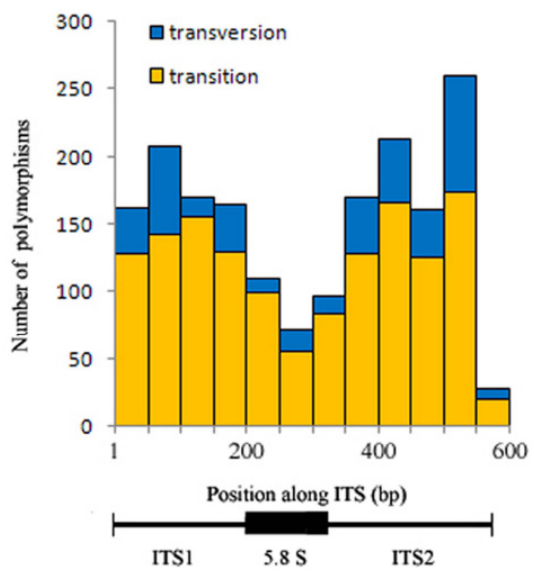

Fig. (3). The distribution of polymorphisms (base substitution-only) in the Rheum tanguticum rDNA array, as derived from ITS sequencing data and shown in 50-bp bins.
Sample RTM10 of $R$. tanguticum is a typical representative of the samples (see the Appendix Table S1), and a multiple sequence alignment of five clones of this sample is shown in Fig. (4). There were 105 variable sites between clones, with the majority of variation existing between KF514583 and the three clones KF514579, KF514580, and KF514581. Only one variable site was observed between KF514583 and KF514582 and a small amount of variation was identified among the other three clones.

\section{Analysis of ITS Sequence Variations by Genetic Distance}

We divided the $R$. tanguticum mutations into two groups, containing intra-specific and intra-genomic variations, which were then analyzed using K2P genetic distances. In particular, we looked at the distribution of divergences within the classes at a resolution 0.02 distance units (Fig. 5). Our results showed that the average intra-specific distance was $0.1260 \pm 0.0755$ and that $R$. tanguticum had a relatively high level of sequence divergence, ranging from 0 to 0.3618 . In addition, K2P intra-genomic distances ranged from 0 to 0.3338 within individual $R$. tanguticum specimens (see the Appendix Table S1). These results demonstrate a high level of variation within the ITS sequences of $R$. tanguticum.

\section{Analysis of Phylogenetic Relationships Based on ITS Se- quences Using NJ Trees}

We constructed a NJ tree based on the 102 ITS sequences of $R$. tanguticum as well as the four GenBank sequences from $R$. officinale, $R$. nobile, $R$. rhabarbarum and $F$. esculentum (Polygonaceae) (Fig. 6). The tree was divided into three groups: (1) ten clones from six samples of $R$. tanguticum (green region) that had high similarity to the ITS sequence of $R$. officinale, (2) a second group containing $R$. nobile, and (3) the remaining eighty-seven clones (including the blue, yellow, and red regions, as well as KF514638) that had high similarity to the ITS sequence of $R$. rhabarbarum. Overall, the NJ tree supportedthe hypothesis that the ITS sequences from $R$. tanguticum were polyphyletic. Unexpectedly, five clones of $R$. tanguticum (purple region) clustered most closely with $F$. esculentum.

\section{DISCUSSION}

The present study revealed a high level of intra-specific genetic polymorphisms in the ITS regions of $R$. tanguticum, which was consistent with the molecular evidence provided by ISSR and SSR analysis in previous reports [8, 25, 30]. The high levels of genetic diversity within the population of $R$. tanguticum may be due to several factors. First, mating systems are thought to be one of the most important factors determining genetic diversity in $R$. tanguticum. In the wild, rheum plants are self-incompatible [31], and it has been proven that self-incompatibility is crucial for the maintenance of high levels of genetic variability within many species [32]. Second, the physiological structure of $R$. tanguticum may be another important factor. Indeed, $R$. tanguticum possesses panicles containing numerous small flowers that can produce large amounts of trigonous achenes, which are small, light, and show a high germination rate between plants [33]. Therefore, for developmental reasons, this species may have more opportunity to accumulate genetic 
KF514579 TGTCGAAACC TGCACAAGCA GACAGACCCG CAAACCCGTC TCTAACCCGC CGTCGGGGGG ATCGGGCTCT CTTTTGAGCC $80 \mathrm{bp}$

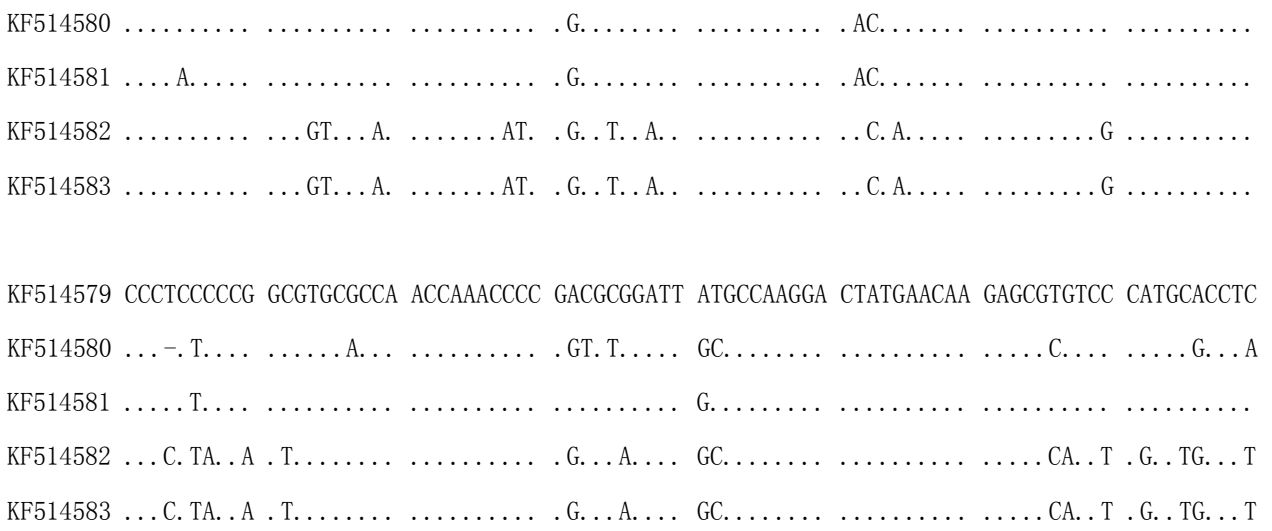

KF514579 GGCGCGCGTG CGACATTGCA TCGTTTCTAC TTAACAGAAC GACTCTCGAC AATGGATATC TTGGCTCTCG CATCGATGAA $240 \mathrm{bp}$

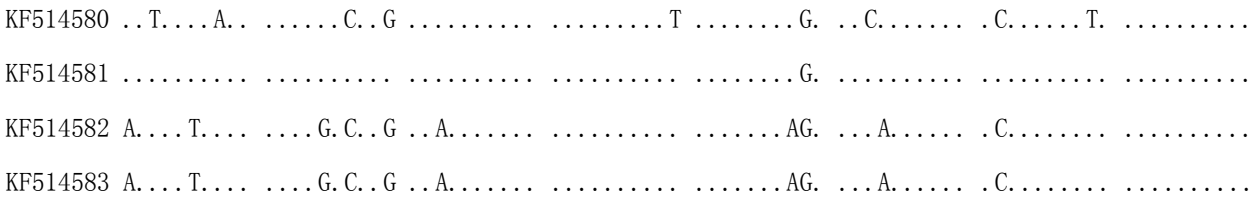
KF514579 GAACGTAGCG AAATGCGATA CTTGGTGTGA ATTGCAGAAT CCCATGAACC ATCGAGTCTT TGAACGCAAG TTGTGCCCGA $320 \mathrm{bp}$

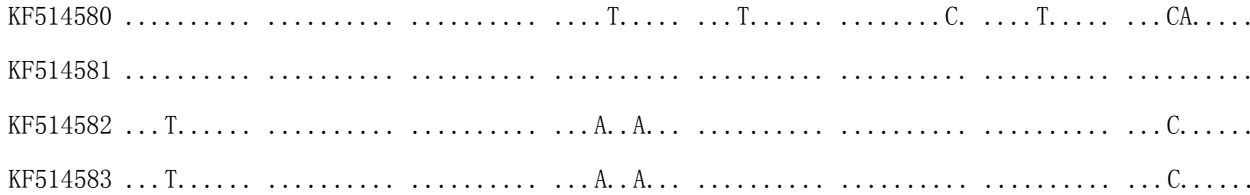
KF514579 AGCCTTCTGG CCGAGGGCAC GTCTGTCTGG GCGTCACGCA CCTTATCGCA CATGCCCCCT CCGAGGGGCC GAGGCAGAGA 400bp

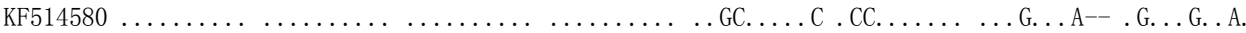
KF514581 .. G..............

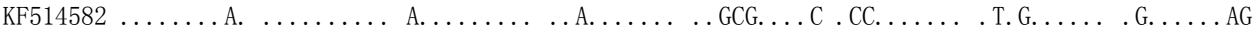
KF514579 CTGGCCTCCC ATGCGTCTCG GTGCACGACC GGCCTAAACG CAGGCCCCGC GGCCACGAGA AGCCGCAACG ATTGGTGGTG 480bp

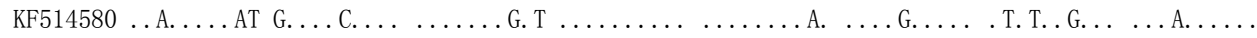
KF51458 . T

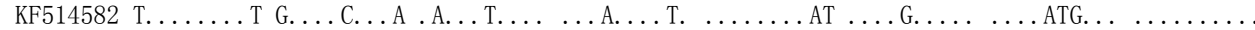

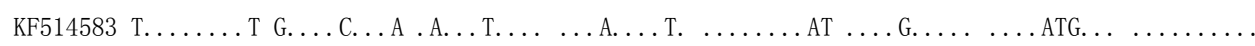
KF514579 TACCAGCGGC CTCGTCCCGT GAAGCATCGC ATCGTGTCTC GCGCGGACCC TGGGCGCCAA AGGGCCTCGA CCACCGTTG 559bp

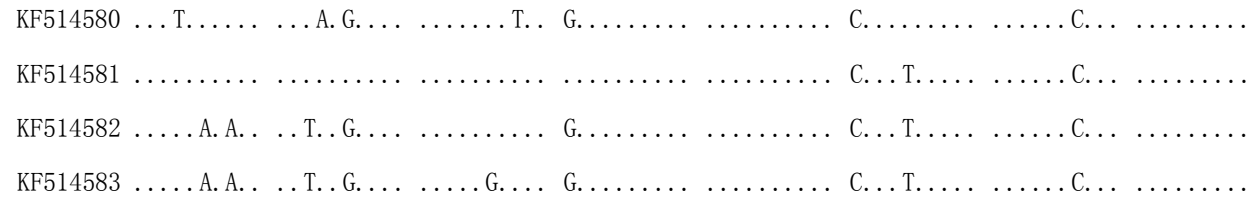

Fig. (4). The complete alignment matrix of the ITS sequence from sample RTM10 of Rheum tanguticum. 


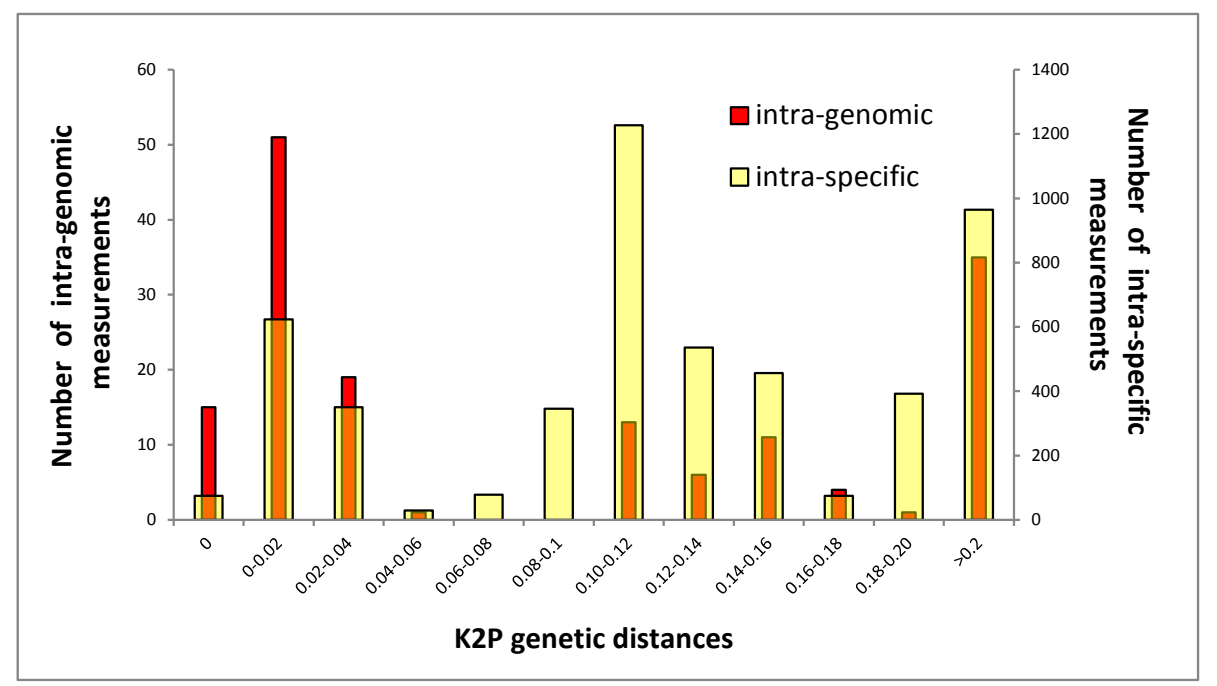

Fig. (5). The relative distributions of intra-genomic divergence(red) between clones within individual Rheum tanguticum samples and intraspecific variation (yellow) for the ITS sequences. $\mathrm{x}$ axis, K2P genetic distance; $y$ axis, frequency.

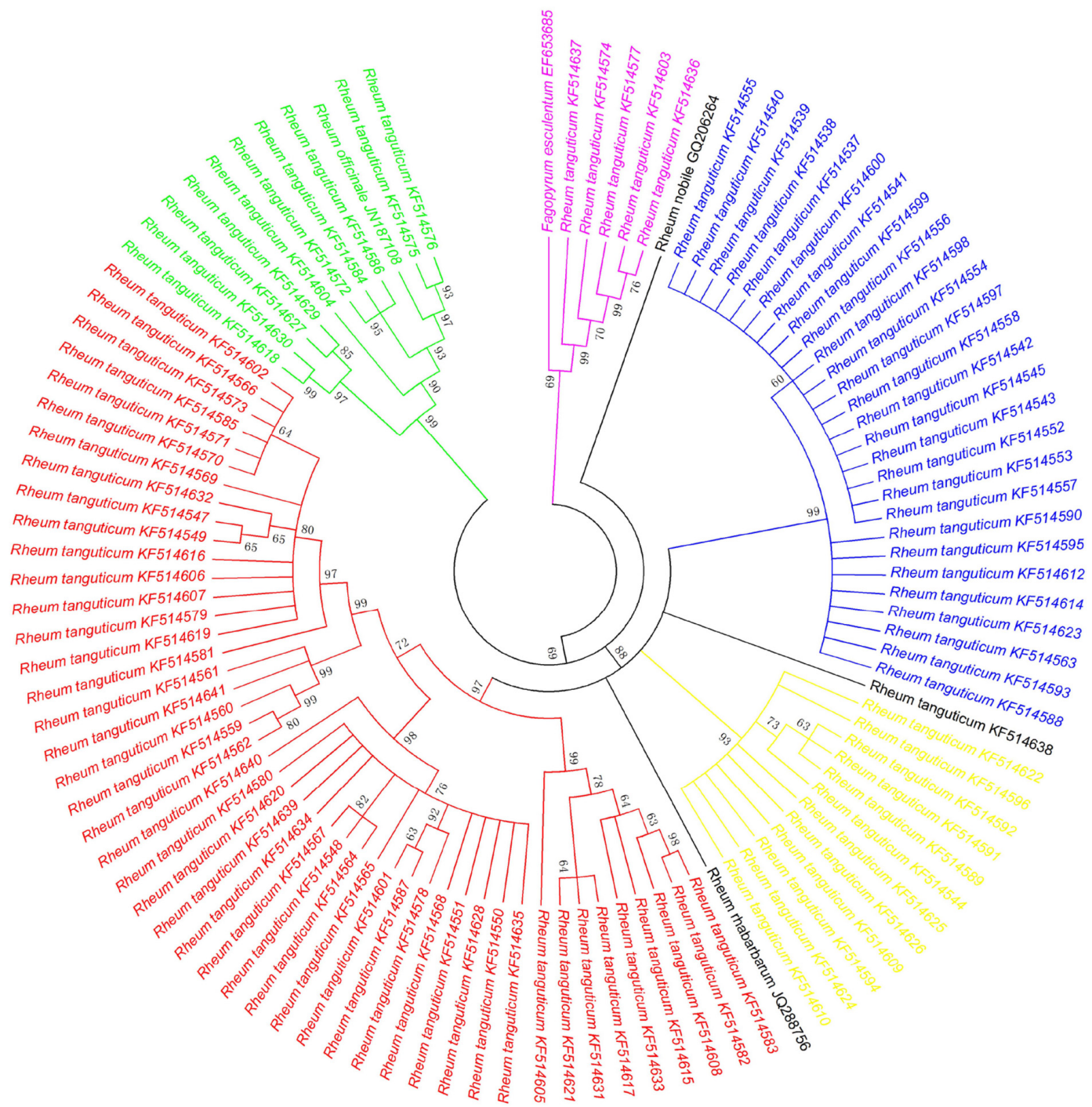

Fig. (6). Circle Neighbor-Joining trees based on the ITS sequences of Rheum tanguticum and several closely related species. Bootstrap scores (1000 replicates) are shown $(\geq 60 \%)$ for each branch. 
mutation compared to other species [34]. Finally, the environment may drive $R$. tanguticum to accumulate high levels of genetic diversity. $R$. tanguticum is distributed primarily throughout the eastern region of the Qinghai-Tibet Plateau [7], which is highly susceptible to climate change. For example, Quaternary climatic oscillations during glacial and interglacial stages in this region may have driven periods of retreat and expansion in this species.

In addition, we captured some genetic information from $R$. tanguticum that had not been revealed previously when we studied genetic diversity between clones. From analyses of the phylogenetic tree, ten clones of $R$. tanguticum had high similarity to ITS sequence of $R$. officinale, which is supported by morphological classification [35]. However, eighty-seven clones had high similarity to ITS sequence of $R$. rhabarbarum, which was consistent with palynological research [36, 37]. Pollen morphology demonstrated that $R$. tanguticum and $R$. rhabarbarum were both microechinatefoveolate, while $R$. nobile and $R$. officinale were different. Unexpectedly, five clones of $R$. tanguticum were similar to $F$. esculentum. These two species belong to different genera of the Polygonaceae, which may indicate that the ITS of nrDNA inherited from parents can reveal horizontal genetransfer and hybridization events in the species evolutionary history. The phenomena of identical variants across genera in nature were also found in the nuclear genome of Eleutherococcus giraldii and Panax ginseng of Araliaceae [38] and in the nuclear genome of Dendropanax hainanensis and Merillinopanax membranifolius of Araliaceae [39].

We suggest that the preferable choice for protection of $R$. tanguticum is in situ conservation because of its high intraspecific genetic variation and its living environment. Genetic diversity is important to consider when formulating conservation strategies for species because it is critical to the capability of a species to adapt to environmental changes [40]. In other words, we must select appropriate protection methods to maintain as much genetic diversity as possible. For example, due to low intra-specific variation and fewer wild resources of $P$. ginseng, tissue culture, somatic embryogenesis, and cultivation were applied [41-43]. However, high genetic variation and highly structured geographic patterns were found in Lamiophlomis rotate (Lamiaceae), so in situ method was used to preserve it in as many populations as possible [44]. In addition, we also consider the physiological characteristics and the living conditions of species when formulating conservation strategies. The protection of Eucalyptus impensa in Western Australia is a classic example. After in situ conservation, this species was still threatened due to frequent fire and disease. Additionally, the seeds of this species were difficult to obtain, thus tissue culture and micropropagation methods, such as in vitro methods, were used in the propagation of E. impensa and had success [45]. Nevertheless, over-exploitation has severely threatened the availability of $R$. tanguticum in nature. Hence, based on the high genetic diversity of $R$. tanguticum, the first step will be to prohibit excavation and establish nature reserves for remaining populations. The second step will be to conduct artificial cultivation to breed excellent germplasms of $R$. tanguticum and solve the difference between conservation of $R$. tanguticum and increasing market requirements.

\section{CONCLUSIONS}

In summary, the present study revealed a high level of genetic diversity in the ITS regions of $R$. tanguticum. In addition, we identified genetic information from $R$. tanguticum that has not been revealed previously. Our results showed that ten clones of $R$. tanguticum had high similarity to the ITS sequence of $R$. officinale, eighty-seven clones had high similarity to the ITS sequence of $R$. rhabarbarum, and five clones of $R$. tanguticum were similar to $F$. esculentum. Based on these results, in situ conservation is the preferable choice for protection of R. tanguticum.

\section{CONFLICT OF INTEREST}

The author(s) confirm that this article content has no conflicts of interest.

\section{ACKNOWLEDGEMENT}

We are thankful for the great support and friendly cooperation from China Meheco Co., Ltd, the Botanical Raw Material Research Department, TSUMURA \& $\mathrm{CO}$ and $R$. tanguticum Research Establishment and Farm of Baihe Pasture, Zoige County, Sichuan Province. This work was supported by the Conservation on Natural Resources of Rhubarb , Program for Innovative Research Team in IMPLAD, and the National Natural Science Foundation of China Project (Grant no. 81373922).

$$
\begin{aligned}
& \text { ABBREVIATIONS } \\
& \text { ITS = Internal transcribed spacer of nuclear ribo- }
\end{aligned}
$$

\section{REFERENCES}

[1] Liu, L.; Yuan, S.F.; Long, Y.; Guo, Z.J.; Sun, Y.; Li, Y.H.; Niu, Y.B.; Li, C.; Mei, Q.B. Immunomodulation of Rheum tanguticum polysaccharide (RTP) on the immunosuppressive effects of dexamethasone (DEX) on the treatment of colitis in rats induced by 2,4,6-trinitrobenzene sulfonic acid. Int. Immunopharmacol., 2009, 9 (13-14), 1568-1577.

[2] Krenn, L.; Presser, A.; Pradhan, R.; Bahr, B.; Paper, D.H.; Mayer, K.K.; Kopp, B. Sulfemodin 8-O-B-D-Glucoside, a new sulfated anthochinone glycoside, and antioxidant phenolic compounds from Rheum emodi. J. Nat. Prod., 2003, 66 (8), 1107-1109. 
[3] Chinese Pharmacopoeia Commission. Pharmacopoeia of the People's Republic of China; China Medical Science and Technology Press: Beijing 2010

[4] Huang, Q.; Shen, H.M.; Shui, G.H.; Wenk, M.R.; Nam Ong, C. Emodin Inhibits Tumor Cell Adhesion through Disruption of the Membrane Lipid Raft-Associated Integrin Signaling Pathway. Cancer. Res., 2006, 66 (11), 5807-5815.

[5] Srinivas, G.; Anto, R.J.; Srinivas, P.; Vidhyalakshmi, S.; Senan, V.P.; Karunagaran, D. Emodin induces apoptosis of human cervical cancer cells through poly (ADP-ribose) polymerase cleavage and activation of caspase-9. Eur. J. Pharmacol., 2003, 473 (2-3), 117125

[6] Liu, S.W. Flora of Qinghai. Qinghai People Press: Xining, 1997, pp. $155-156$.

[7] Bao, B.J.; Grabovskaya-Borodina, A.E. Flora of China. Li, A.R.; Bao, B.J., Ed.; Science Press: Beijing, Missouri Botanical Garden: St. Louis, 2003, 5, pp. 341-350.

[8] Hu, Y.P.; Wang, L.; Xie, X.L.; Yang, J.; Li, Y.; Zhang, H.J. Genetic diversity of wild populations of Rheum tanguticum endemic to China as revealed by ISSR analysis. Biochem. Syst. Ecol, 2010, 38 (3), 264-274

[9] Jiao, X.L.; Zhang, F.L. Cultivation methods of Rheum tanguticum in Guoluo. China Sci. Technol. Inform., 2006, 5, 116.

[10] Cote, C.T. Genetic variation in rare and common plants. Annu. Rev. Ecol. Syst., 2003, 34, 213-237.

[11] Huang, Y.; Zhang, C.Q.; Li, D.Z. Low genetic diversity and high genetic differentiation in the critically endangered Omphalogramma souliei (Primulaceae): implications for its conservation. J. Syst. Evol., 2009, 47 (2), 103-109.

[12] Torres, E.; Iriondo, J.M.; Escudero, A.; Pérez, C. Analysis of within-population spatial genetic structure in Antirrhinum microphyllum (Scrophulariaceae). Am. J. Bot., 2003, 90(12), 1688-1695.

[13] de Jesus, O.N.; Silva, Sde.O.; Amorim, E.P.; Ferreira, C.F.; de Campos, J.M.; Silva, Gde.G.; Figueira, A. Genetic diversity and population structure of Musa accessions in ex situ conservation. BMC Plant Biol., 2013, 13, 41.

[14] Tero, N.; Aspi, J.; Siikamäki, P.; Jäkäläniemi, A.; Tuomi, J. Genetic structure and gene flow in a metapopulation of an endangered plant species, Silene tatarica. Mol. Ecol., 2003, 12 (8), 2073-2085.

[15] Ding, G.; Zhang, D.Z.; Ding, X.Y.; Zhou, Q.; Zhang, W.C.; Li, X.X. Genetic variation and conservation of the endangered Chinese endemic herb Dendrobium officinale based on SRAP analysis. Plant Syst. Evol., 2008, 276, 149-156.

[16] Chinese Plant BOL Group. Comparative analysis of a large dataset indicates that internal transcribed spacer (ITS) should be incorporated into the core barcode for seed plants. Proc. Nat. Acad. Sci. USA, 2011, 108 (49), 19641-19646.

[17] Hřibová, E.; Č́ižková, J.; Christelová, P.; Taudien, S.; de Langhe, E.; Doležel, J. The ITS1-5.8S-ITS2 sequence region in the Musaceae: structure, diversity and use in molecular phylogeny. PLoS One, 2011, 6 (3), e17863.

[18] Nieto Feliner, G.; Rosselló, J.A. Better the devil you know? Guidelines for insightful utilization of nrDNA ITS in species-level evolutionary studies in plants. Mol. Phylogenet. Evol., 2007, 44 (2), 911919.

[19] Chen, S.L.; Yao, H.; Han, J.P.; Liu, C.; Song, J.Y.; Shi, L.C.; Zhu, Y.J.; Ma, X.Y.; Gao, T.; Pang, X.H.; Luo, K.; Li, Y.; Li, X.W.; Leon, C. Validation of the ITS2 region as a novel DNA barcode for identifying medicinal plant species. PLoS One, 2010, 5 (1), e8613.

[20] Chase, M.W.; Fay, M.F. Barcoding of plants and fungi. Science, 2009, 325, 682-683.

[21] Poczai, P., Hyvönen, J. Nuclear ribosomal spacer regions in plant phylogenetics: problems and prospects. Mol. Biol. Rep., 2010, 37 (4), 1897-1912.

[22] Yamaji, H.; Fukuda, T.; Yokoyama, J.; Pakd, J.H.; Zhou, C.Z.; Yang, C.S.; Kondo, K.; Morota, T.; Takeda, S.; Sasaki, H.; Maki, M. Reticulate evolution and phylogeography in Asarum sect. Asiasarum (Aristolochiaceae) documented in internal transcribed spacer sequences (ITS) of nuclear ribosomal DNA. Mol. Phylogenet. Evol., 2007, 44 (2), 863-884.

[23] Matsumura, S.; Yokoyama, J.; Fukuda, T.; Maki, M. Intraspecific differentiation of Limonium wrightii (Plumbaginaeae) on northwestern Pacific Islands: Rate heterogeneity in nuclear rDNA and its distance-independent geographic structure. Mol. Phylogenet. Evol., 2009, 53 (3), 1032-1036.

[24] Sass, C.; Little, D.P.; Stevenson, D.W.; Specht, C.D. DNABarcoding in the Cycadales: Testing the Potential of Proposed Barcoding Markers for Species Identification of Cycads. PLoS One, 2007, 2 (11), e1154.

[25] Chen, F.J.; Wang, A.L.; Chen, K.M.; Wan, D.S.; Liu, J.Q. Genetic diversity and population structure of the endangered and medically important Rheum tanguticum (Polygonaceae) revealed by SSR markers. Biochem. Syst. Ecol., 2009, 37 (5), 613-621.

[26] White, T.J.; Bruns, T.; Lee, S.; Taylor, J. Amplification and direct sequencing of fungal ribosomal RNA genes for phylogenetics. Innis, M.A.; Gelfand, D.H.; Sninsky, J.J.; White, T.J., Ed.; Academic: San Diego, 1990; pp. 315-322.

[27] Kress, W.J.; Wurdack, K.J.; Zimmer, E.A.; Weigt, L.A.; Janzen, D.H. Use of DNA barcodes to identify flowering plants. Proc. Natl. Acad. Sci. USA, 2005, 102 (23), 8369-8374.

[28] Tamura, K.; Peterson, D.; Peterson, N.; Stecher, G.; Nei, M.; Kumar, S. MEGA5: molecular evolutionary genetics analysis using maximum likelihood, evolutionary distance, and maximum parsimony methods. Mol. Biol. Evol., 2011, 28 (10), 2731-2739.

[29] Keller, A.; Schleicher, T.; Schultz, J.; Müller, T.; Dandekar, T.; Wolf, M. 5.8S-28S rRNA interaction and HMM-based ITS2 annotation. Gene, 2009, 430 (1-2), 50-57.

[30] Wang, X.M.; Yang, R.; Feng, S.F.; Hou, X.Q.; Zhang, Y.Q.; Li, Y.; Ren, Y. Genetic Variation in Rheum palmatum and Rheum tanguticum (Polygonaceae), Two Medicinally and Endemic Species in China Using ISSR Markers. PLoS One, 2012, 7 (12), e51667.

[31] Komatsu, K.; Nagayama, Y.; Tanaka, K.; Ling, Y.; Basnet, P.; Meselhy, M.R. Development of a high performance liquid chromatographic method for systematic quantitative analysis of chemical constituents in rhubarb. Chem. Pharm. Bull., 2006a, 54 (7), 941947.

[32] Borba, E.L.; Semir, J.; Shepherd, G.J. Self-incompatibility, inbreeding depression and crossing potential in five Brazilian Pleurothallis (Orchidaceae) species. Ann. Bot., 2001, 88, 89-99.

[33] Xiao, S.P.; Chen, M.; Huang, L.Q.; Gao, F. Primary study on shapes of fruits and germination characters of seeds of Radix et Rhizoma Rhei. China J. Chin. Mater. Med., 2007, 32 (3), 195-199.

[34] Nybom, H. Comparison of different nuclear DNA markers for estimating intraspecific genetic diversity in plants. Mol. Ecol., 2004, 13 (5), 1143-1155.

[35] Li, A.R. Flora Republicae Popularis Sinicae; Science Press: Beijing, 1998.

[36] Wang, A.L.; Yang, M.H.; Liu, J.Q. Molecular phylogeny, recent radiation andevolution of gross morphology of the rhubarb genus Rheum (Polygonaceae) inferred from chloroplast DNA trnL-F sequences. Ann. Bot., 2005, 96, 489-498.

[37] Yang, M.H.; Zhang, D.M.; Zheng, J.H.; Liu, J.Q. Pollen morphologyand its systematic and ecological significance in Rheum (the Rhuburb genus, Polygonaceae) from China. Nord. J. Bot., 2001, 21, 411-418.

[38] Song, J.Y.; Shi, L.C.; Li, D.Z.; Sun, Y.Z.; Niu, Y.Y.; Chen, Z.D.; Luo, H.M.; Pang, X.H.; Sun, Z.Y.; Liu, C.; Lv, A.; Deng, Y.P.; Larson-Rabin, Z.; Wilkinson, M.; Chen, S.L. Extensive pyrosequencing reveals frequent intra-genomic variations of internal transcribed spacer regions of nuclear ribosomal DNA. PLoS One, 2012, 7 (8), e43971.

[39] Mitchell, A.; Wen, J. Phylogeny of Brassaiopsis (Araliaceae) in Asia based on nuclear ITS and 5S-NTS DNA sequences. Syst. Bot., 2005, 30, 872-886.

[40] Jump, A.S.; Marchant, R.; Peñuelas, J. Environmental change and the option value of genetic diversity. Trends Plant Sci., 2009, 14 (1), 51-58.

[41] Kevers, C.; Gal, N.L.; Monteiro, M.; Dommes, J.; Gaspar, T. Somatic embryogenesis of Panax ginseng in liquid cultures: a role for polyamines and their metabolic pathways. J. Plant Growth Regul., 2000, 31, 209-214.

[42] Kim, Y.J.; Kim, M.K.; Shim, J.S.; Pulla, R.K.; Yang, D.C. Somatic embryogenesis of two new Panax ginseng cultivars Yun-Poong and Chun-Poong. Russian J. Plant Physiol., 2010, 57, 283-289.

[43] Chen, X.C.; Liao, B.S.; Song, J.Y.; Pang, X.H.; Han, J.P.; Chen, S.L. A fast SNP identification and analysis of intraspecific varia- 
tion in the medicinal Panax species based on DNA barcoding. Gene, 2013, 530, 39-43.

[44] Liu, J.; Wang, L.; Geng, Y.; Wang, Q.; Luo, L.; Zhong, Y. Genetic diversity and population structure of Lamiophlomis rotate (Lamiaceae), an endemic species of Qinghai-Tibet Plateau. Genetica, 2006, $128(1-3), 385-94$.
[45] Bunn, E. Development of in vitro methods for ex situ conservation of Eucalyptus impensa, an endangered mallee from southwest Western Australia. Plant Cell Tissue Organ Cult., 2005, 83, 97102.

Received: August 28, 2013

Revised: December 16, 2013

Accepted: December 18, 2013

(C) Ma et al.; Licensee Bentham Open.

This is an open access article licensed under the terms of the Creative Commons Attribution Non-Commercial License (http://creativecommons.org/licenses/by-nc/3.0/) which permits unrestricted, non-commercial use, distribution and reproduction in any medium, provided the work is properly cited. 